\title{
Virtual Sculpting and 3D Printing for Young People with Disabilities
}

\author{
Leigh MCLOUGHLIN ${ }^{a^{*}}$, Oleg FRYAZINOV ${ }^{\mathrm{a}}$, Mark MOSELEY ${ }^{\mathrm{b}}$, Mathieu SANCHEZ, \\ Valery ADZHIEV ${ }^{\mathrm{a}}$, Peter COMNINOS ${ }^{\mathrm{a}}$, Alexander PASKO ${ }^{\mathrm{a}}$ \\ ${ }^{\mathrm{a} N C C A}$, Bournemouth University, $U K$ \\ ${ }^{b}$ Victoria Education Centre, UK \\ *Imcloughlin@bournemouth.ac.uk
}

\begin{abstract}
In this paper, we present the SHIVA project which was designed to provide virtual sculpting tools for young people with complex disabilities, to allow them to engage with artistic and creative activities that they might otherwise never be able to access. Modern 3D printing then allows us to physically build their creations. To achieve this, we combined our expertise in education, accessible technology, user interfaces and geometric modelling. We built a generic accessible graphical user interface (GUI) and a suitable geometric modelling system and used these to produce two prototype modelling exercises. These tools were deployed in a school for students with complex disabilities and are now being used for a variety of educational and developmental purposes. In this paper, we present the project's motivations, approach and implementation details together with initial results, including 3D printed objects designed by young people who have disabilties.
\end{abstract}

Keywords: Virtual Sculpting, Accessibility, User Interfaces, Disabilities, 3D Printing

\section{Introduction}

Artistic activities are an important educational subject in their own right and can also provide strong links with other core subjects and life-skills such as spatial awareness, object recognition, as well as aspects such as self-expression and building self-confidence. However, using clay or any other sculpting material is non-trivial for disabled individuals with little or no limb control. In the SHIVA project, we proposed to use computer-based technologies to extend access to such artistic tools for particularly vulnerable population groups: people in rehabilitation (through French partners in University Lille 1 and the HOPALE Foundation) and young people with various types of disabilities (by the authors in the UK at Bournemouth University (BU) and Victoria Education Centre (VEC)). In this paper we present the motivations, approach and initial results of the SHIVA project's use with young people.

Young people with disabilities may have a very different experience of the physical world to those without. This experience may be influenced by their range of movement, coarse or fine motor control, or having spent their life in a wheelchair. Due to these physical difficulties, they may not have had the opportunity to explore the physical properties of different objects and materials in a conventional sense. New technologies are helping to provide ways in which young people who have disabilities can have these experiences in a virtual sense. Thus, the aim of the SHIVA project was to enable such young people to learn about manipulating objects by providing a few basic virtual sculpting tools and then producing the objects physically with 3D printing technologies.

Given these motivations, the primary research objectives were:

1. To consider and establish the ranges of interface requirements needed by young people with disabilities to enable them to use software of varying complexity.

2. To examine how a generic user interface system can be designed to meet these requirements to give access for users with a broad range of physical input requirements.

3. To identify and develop virtual sculpting methods that would be appropriate for the target audience (young people with physical and / or cognitive disabilities) and accessible to them given their input requirements, while also allowing 3D printing for real-world output. 
The current state-of-the-art in accessible technology and virtual sculpting (see the sidebar) includes a range of impressive tools. However, we found that the available virtual sculpting software would not allow us to achieve the goals of the SHIVA project. The team had to develop new solutions for the virtual sculpting based on the group's scalar field or Function Representation (FRep) based modelling system (Pasko et al, 1995) due to its feature set and natural 3D printing suitability. It was also decided that existing accessible user interface systems were unsuitable for the needs of the project, so a new solution was developed.

\section{Accessible Technologies and Virtual Sculpting (sidebar)}

Brief summaries are provided here on the state-of-the-art of both aspects essential to the project: accessible user interface technologies and virtual sculpting.

\section{User Models and Accessible User Interfaces}

User interfaces provide the software interface layer between the human user and the actual software application. Most computers currently support user input from keyboard, mouse, touchscreen and to some extent voice commands while most output modalities rely on information-rich visual displays, which can be difficult for the visually impaired. While some of the project's students can use a mouse or touchscreen, others struggle to press a single large button, or have absolutely no limb control and can only access software through an eye-gaze system.

One of the key aspects of access for disabled users is that each individual has very different and specific interface requirements and that these requirements can vary according to their specific physical or cognitive abilities and that these abilities are dynamic and liable to change, often throughout each day. The user interface must therefore store some information about its users in order to be configured to their requirements. This information is called a user model and can generally be stored in two ways: first is a medical approach, where information about the user's physical capabilities and limitations are stored; and a functional approach, where the user's interface requirements are stored.

To overcome modern operating system limitations, specialist accessible interface toolkits have been produced:

The Grid 2 [http://sensorysoftware.com/grid-software-for-aac/] is a commonly used commercial system within special schools and provides disabled access primarily for communication but also for other features. It has a very deep level of support for communication, such as offering phonetics for synthesised vocal output, but these features require a specialist speech or language therapist to use or set up. The software provides access through switch, touchscreen, eye-gaze, headpointer and mouse / keyboard. This covers a wide range of inputs and features, but is not open or flexible enough for a heavy-weight application such as interactive 3D modelling.

The MyUI project (Peissner et al, 2012) has an adaptive interface but is targeted mostly at elderly people. It presents a set of predefined applications including email access, TV, games and similar consumables. The user profile, which covers vision, hearing, language, information processing, memory, computer skills, speech, dexterity and muscle strength is adjusted while user is working with the system. However as a limited amount of sensors to detect user's abilities are used and only simple data is stored, it is not clear if this data can be used in any practical sense.

The GUIDE project (Jung et al, 2012) has a user-centred approach to create basic UIs with a large variety of input devices as well as accessibility options. The project is mainly aimed at and mostly tested with elderly people and allows simplifying the access to the entertainment (TV) and communication (video calls). The system allows automatic user profile setup by guiding the user through a simple test which determines the cognitive abilities of the user as well as some disabilities of the user such as colour blindness. The input devices for the user interface include tablets, speech, gestures, and eye-gaze. Overall, the main limitations of the system are its orientation to easier access for consumption of information rather than creating new information. 


\section{Virtual Sculpting}

Virtual Sculpting is a computer-aided technology allowing for the creation of sculptural artefacts. It can be performed in various ways: using 2D/3D input, using an interactive modelling technique that employs pressure-sensitive or haptic interactions, or alternatively, using a set of virtual reality interface tools such as cybergloves or Digital Clay.

Virtual Sculpting techniques employ a variety of sculptural metaphors (Adzhiev et al, 2005) such as the construction of 3D shapes using Constructive Solid Geometry (CSG) techniques; global and local deformations that are suitable for disabled users. Interactive local modifications can be done using different sculpting metaphors. The concept of a Virtual Clay (McDonnell et al, 2001) is perhaps the most natural metaphor for virtual sculpting. It is supported by a number of commercial and research products such as Geomagic Freeform and Claytools, Cubify Sculpt, Pixologic's ZBrush and Sculptris.

In the Augmented Sculpture Project by the SHIVA team (Adzhiev et al, 2005), a specific interactive environment with embedded sculptural means was created. The user experiences an immersion into a virtual space where they can generate new shapes using either metamorphosis between several predefined sculpture models or the virtual carving tool with such operations as subtraction, offsetting and blending. Finally the resulting sculpting artefacts were 3D-printed to produce new physical sculptures. The project had both artistic and educational merits (Pasko and Adzhiev, 2009) and the tools and lessons learned fed directly into the SHIVA project.

\section{References (for the side bar)}

Adzhiev V., Comninos P., Kazakov M. \& Pasko A. (2005) Functionally based augmented sculpting. Journal of Visualization and Computer Animation, 16(1), 25-39

Jung C., Hamisu P., Duarte C., Biswas P. \& Almeida L. (2012) GUIDE: Personalisable Multi-modal User modal User Interfaces for Web Applications on TV Interfaces, Proceedings of NEM Summit 2012

McDonnell K. T., Qin H., \& Wlodarczyk R. A. (2001) Virtual clay: a real-time sculpting system with kaptic toolkits, Symposium on Interactive 3D Graphics, ACM, 2001, pp. 179-290.

A. Pasko, V. Adzhiev. (2009) Constructive function-based modeling in multilevel education, Communications of the ACM, Vol. 52, No. 9, pp. 118-122.

Peissner M., Häbe D., Janssen D. \& Sellner T. (2012) MyUI: Generating accessible user interfaces from multimodal design patterns. In: Proceedings of the 4th ACM SIGCHI Symposium on Engineering Interactive Computing Systems EICS '12 (pp 81-90), Copenhagen, Denmark: ACM Press.

\section{Our approach}

The core of the project is interactive applications allowing the users to manipulate the virtual objects in order to create new shapes in the 3D environment. In all the software applications we can distinguish two main components: an accessible graphical user interface and the interactive solid modelling system that allows the manipulation of geometric shapes.

\section{Accessible Graphical User Interface (GUI)}

To achieve the research objectives of the project and to overcome the limitations of the current stateof-the-art, new interface tools needed to be developed to allow each disabled user to interact with the software. An interface solution must therefore provide a vast range of flexibility and the ability to store settings for each user.

For this user modelling, a functional approach was determined the most suitable because it directly maps the user's interface needs with the software settings. The choice of input mode and the specific settings would be stored in a user profile which was to be created for each individual student to meet their needs.

The user's physical needs were gradually identified and discussed, often using simple test applications, in a way that focussed on the direct requirements for the interface and identified the required features and their ranges. This informed the development of a generic graphical user interface (GUI) system which could be used to map all sculpting features to on-screen 'buttons'. This 
provides a lowest common denominator from which all interface devices and modalities could be successfully employed. For single button access the interface used switch-scanning (Colven \& Judge 2006), where each GUI button is highlighted in turn until the user presses their switch to select the current element. The use of switch scanning allows an interactive system to work with a broad range of physical user interface technologies ranging from gaze/blink systems, to sip-and-puff tubes, finger, foot, or head motions, and others, which are adaptable for users across a broad range of disabilities.

The final SHIVA GUI system features include: switch-scanning support with adjustable timing parameters, direct progression with multiple switches; mouse or touchscreen control; button debouncing options; key-mapping options with activation on trailing or leading edges; basic eye-gaze support with adjustable dwell time and configurable rest zones; fully configurable GUI layouts which can be saved and loaded from user profile; visual styling in themes for use across multiple profiles; visual adjustment in themes and profiles; configurable graphics for buttons, symbols, text, including sophisticated colour replacement in graphics.

\section{Shape Modelling System Core}

Another project objective was to allow the users to sculpt virtual objects, i.e. perform operations on geometric shapes in a virtual environment. It is clear that to make the modelling core of the system extensible, the layout that works with representation and manipulations of the geometry should be universal. In our system we represent geometry in the implicit form by using the Function Representation (Pasko et al, 1995). FRep represents geometric objects using continuous real functions over a set of coordinates. This representation allows us to describe a vast number of geometric primitives and perform lots of operations in the solid modelling system in a more simple and efficient way compared to other traditional representations, such as polygonal meshes. Easy formulation allows us to work with traditional geometric primitives such as a sphere, box and cylinder. Beyond this, more complex geometric primitives such as polygonal meshes can be represented efficiently in the form of signed distance fields (Sanchez et al, 2012) which is a natural subset of FRep. Traditionally, the main disadvantage of such a representation is that the geometry of the object cannot be rendered using the common software for standard graphics hardware. Instead, in our system we use direct rendering of the object in the form of real-time ray-casting of objects through acceleration with graphics hardware (Fryazinov and Pasko, 2008).

The objects and operations are represented in the form of a tree that generates the defining function for the model. In the leaves of such a tree we have the geometric primitives and objects, while in the nodes we have operations over other nodes and leaves (Pasko and Adzhiev, 2004). This allows us to perform operations in the modelling system by modifying the structure of the tree itself by adding and removing nodes. Existing models are fully parameterised by modifying the values for the parameters of primitives and operations.

As an intermediate format for the geometry we are using the volumetric object format developed by the Norwegian company Uformia. This supports most of the operations and primitives existing in the current state of the art in modelling with geometry represented in an implicit form. This format allows the interchange of models between applications, using them as the source for direct fabrication and also converting these models to other formats for further operations in other modelling systems and applications. For example, most 3D printing hardware takes only polygonal meshes as an input, therefore we must convert the model from implicit form to a polygonal mesh by using polygonization methods. 


\section{Applications}

Two exercise applications have been developed within the SHIVA project, namely Metamorphosis and Totem Pole, with different levels of complexity on both user interface and geometric modelling sides.

\section{Metamorphosis Exercise}

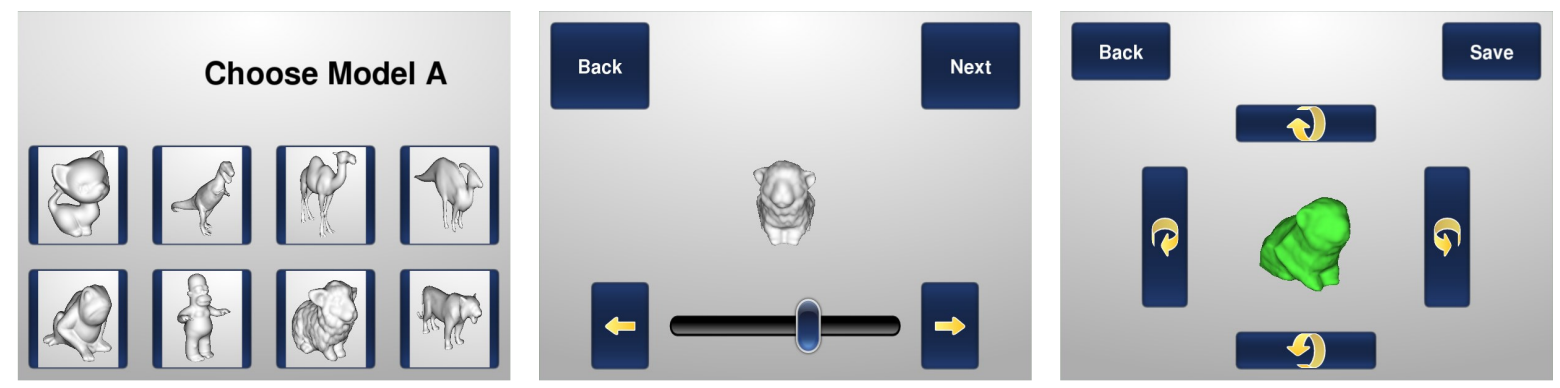

Figure 1. The metamorphosis exercise: the student chooses two models, can make a morph between them, then rotate and colour the resulting shape. Here, a morph between a sheep and a frog model is selected with an intermediate shape seen at the right.

The first prototype software using the accessible GUI was a metamorphosis exercise, specifically for younger or less cognitively able students. Here, the user chooses two objects and can produce an intermediate shape that is blend between the two objects. Primary interaction for this is through a slider and the blended shape is displayed to the user and updated interactively. The user can then rotate their object and apply a colour to it.

As we discussed above, two main components of all our applications are an accessible GUI system and geometric representation of the objects with scalar fields (or FRep). The interface of the metamorphosis application is shown in Fig. 1. It can be seen that the interface is implemented in a simplistic way allowing even a user with severe disabilities to interactively perform the transition operation between two shapes.

From the geometric point of view, in the application we perform metamorphosis operations over two FRep objects. Given the nature of the application, we used polygonal meshes of existing real-world objects converted to scalar fields as sources for the input shapes. The metamorphosis operation in its simplest form can be seen as a linear interpolation between the values of the scalar field for the initial object and the target object. Also we have tried more complex metamorphosis operations (Sanchez et al, 2013) such that the user can influence the process in order to obtain more artist-friendly intermediate shapes.

The output of the application is the solid object representing the intermediate stage of the metamorphosis between two objects. As the object is solid, it can be further used as an input object for the application or used as an input for 3D printing.

\section{Totem Pole Exercise}
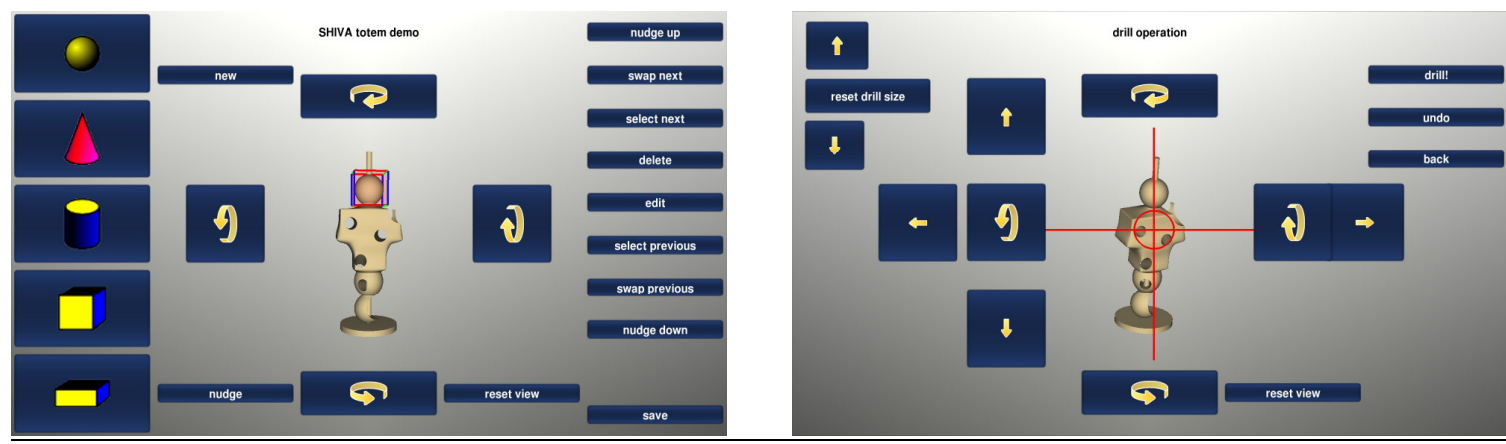
Figure 2. The totem-pole exercise interface. Left: main construction screen with buttons to add primitive geometric shapes to the stack, navigation buttons and a designed shape showing primitive stacking, blending and drilling. Right: drill operation screen with cross-hair controls for drill location and controls to rotate the object.

The second software prototype was a 'totem-pole' exercise, which provides a more complex sculpting environment. Here, the user stacks a small number of objects together and then performs simple modelling operations such as affine transformations on individual objects within the stack, or operations such as blending and drilling on the entire stack. For this application the accessible UI is more advanced (Fig. 2) compared to the metamorphosis application because more operations are supported and more input is required from the user. However the general approach remains the same.

In this application we allow the user to choose from a number of simple geometric shapes as an input. In the current implementation these shapes include: sphere, box, cylinder and cone. The set of operations over these objects include: Boolean operations (union, and subtraction), smooth union blending between objects, and affine transformations. From the implementation point of view the operations are achieved by modifying the tree representing the object.

As an example, for the drill operation (UI shown in Fig. 2, right) the following steps are performed on the existing tree in order to modify the object based on the user's input:

1. The parameters for the drill are obtained from the camera position and direction and from the predefined (or set by the user from the GUI) diameter. These parameters define a cylinder and a parent affine transformation node that gives it the correct orientation.

2. A node describing a Boolean subtraction is added to the tree. The branches of this node are the cylinder's transform node from step 1 and the top node for the model.

3. The top node for the model becomes the Boolean node added in the previous step.

Thus, adding objects to the stack are Boolean union operations with affine transform nodes giving each object an offset position. Orientation operations on each object then adjust the parameters of this transform node and blending is achieved with a smooth-blended union instead. As we discussed above, the easy definition of the geometric objects and of the operations allows us to create a simple definition of the objects and therefore use these as an input for a direct rendering system in the form of real-time ray casting for interactive visualisation of the intermediate stages of the modelling process.

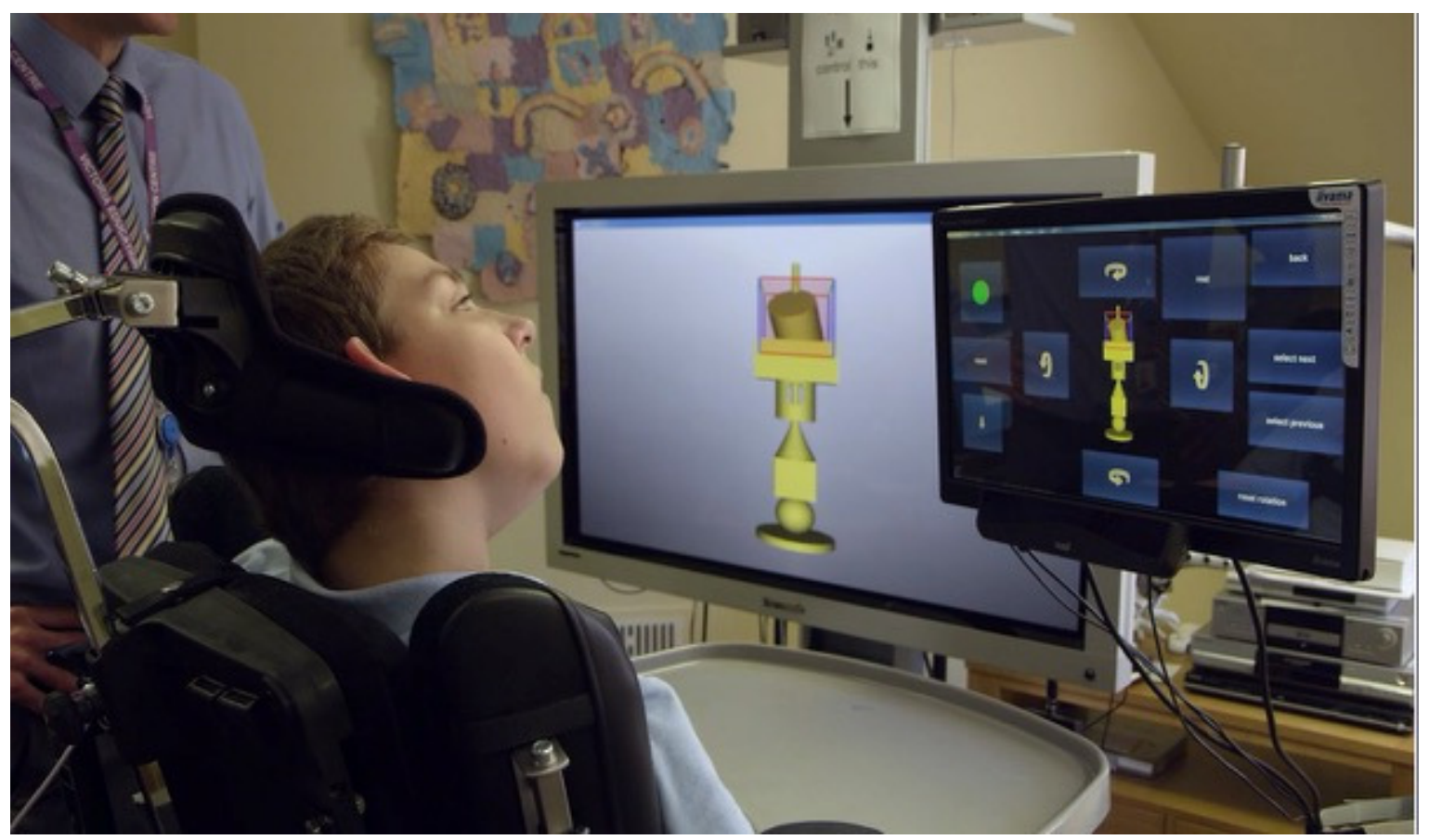

Figure 3. A VEC student using the 'totem-pole' exercise with the eye-gaze control. 


\section{Results}

The prototype software implementations were installed at the Victoria Education Centre (VEC) and as far have been used by 11 students with disabilities including two eye-gaze users (see Fig. 3). During the active development phase (2013), this gave an opportunity for feedback from actual users which was vital for refining the GUI system and software prototypes. Since then, the software has been used by teachers and speech therapists with a variety of students, leading to actual 3D printed results. In each case, the student was under the close supervision and guidance of an assistive technologist in addition to relevant educators and speech therapists.

\section{$3 D$ Printed Sculptures}

The primary technical goal of the SHIVA project was to allow users with disabilties to create virtual and then 3D printed sculptural artefacts. Students were able to successfully produce a range of objects with the system, thereby validating the software and the process. Some results are shown in Fig. 4.

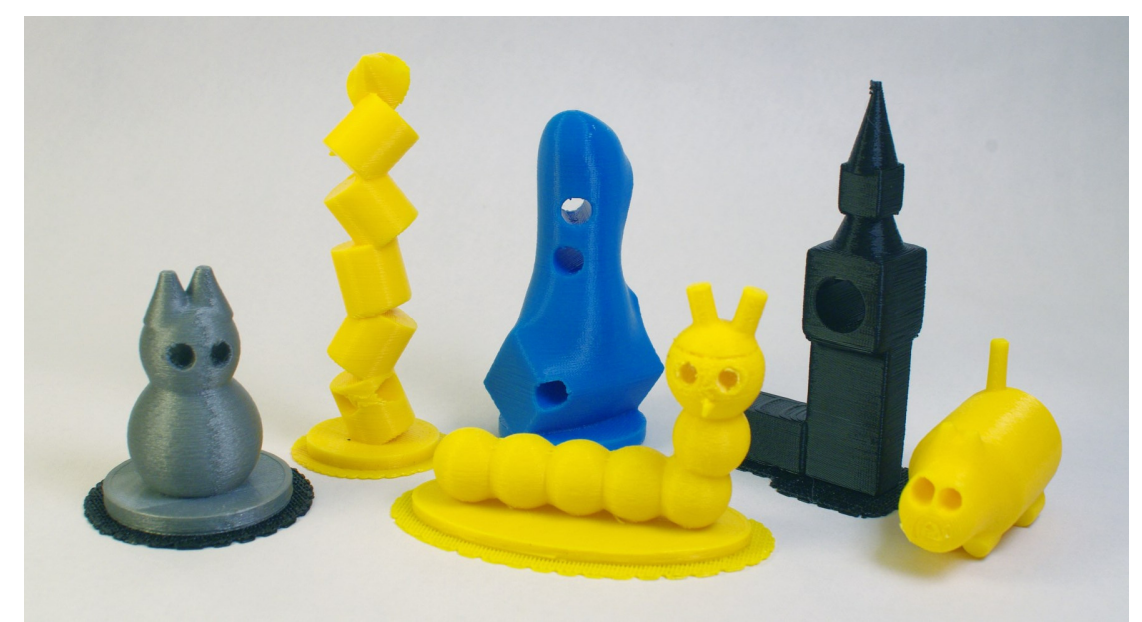

Figure 4. Designs produced using the SHIVA totem-pole exercise and printed at the Victoria Education Centre on a BFB 3D printer. The pupils used largely touch-screen interaction or eye-gaze tracking.

\section{Educational Integration}

Some of the more able students were invited to try the software during the development phase. This was introduced to them in a careful and controlled manner because their reaction to potentially unstable prototype software was unknown. In reality, the students were extremely enthusiastic and took particular delight in the discovery of software bugs.

The SHIVA software has been successfully used to help teach students about spatial relationships between objects and general spatial awareness. It has been used to teach and reinforce spatial concepts such as 'up' and 'down', 'behind', 'rotate' and so on. This has also helped the teachers to understand how young people who have severe physical difficulties perceive certain concepts. The SHIVA Totem software was used by two eye-gaze users and it was discovered that they both had similar difficulties with the concept of a 'stack'. Teachers used a physical stack of primitive objects and asked the users to reproduce the stack using the software. In the SHIVA Totem software objects are added in a stack which starts from the bottom, adding one object on top of the other. However, both eye-gaze users would consistently try to start the stack from the topmost shape first. It is expected that the reason for this is because these users have simply never had the experience of physically placing one object upon another, so the concept is quite new to them. The identification of this has then helped the teachers in their understanding of which fundamental spatial concepts need to be introduced to such students and in assessing their comprehension. 
The software is frequently used to help students understand how the shape of an object may be constructed from a set of simple primitive shapes and understanding the differences between and representations of 2D and 3D shapes. A teacher will often draw an idea on a sheet of paper and then ask the student to reconstruct it using the SHIVA software, giving instructions and answering questions during the process. The teaching approach has been on enjoying the experience, rather than necessarily producing a 'correct' end result. However, students have already shown progress - one student who started with creating essentially random objects is now able to create identifiable models of simple objects such as a cat or a teddy-bear.

Speech therapists at VEC have successfully used the software with students in their regular activities to help with speaking and listening as well as cognitive development aspects. They work with the students on the concepts of sequencing, following instructions, communicating ideas and collaborative work. Early observations suggest this approach will lead to good results for the students, especially due to the high levels of engagement with the software.
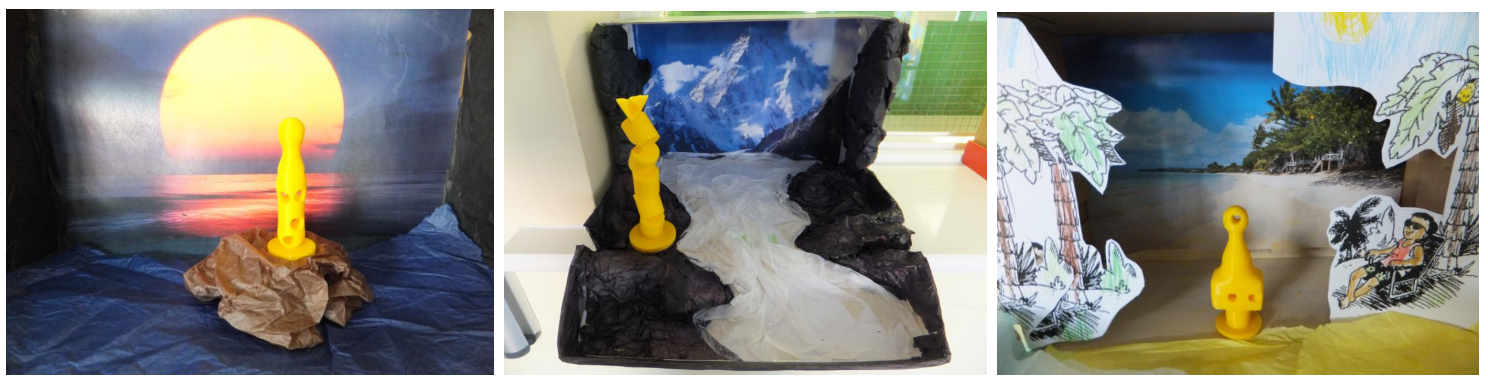

Figure 5. Examples of the 'shoe-box landscape' project inspired by the work of artist Andy Goldsworthy.

The SHIVA software has started to be incorporated into regular scheduled art lessons at VEC, currently in use related to a 'shoe-box landscape' project inspired by the work of British sculptor Andy Goldsworthy (see Fig. 5). This will involve students creating sculptural arches as viewing windows similar to some of his famous works. It is expect that this will be well received by students and lead to increased engagement with the project.

Physical benefits are also being investigated. Therapists are starting to use the software as a tool from the point of view of an aid in improving manual dexterity, or through a touch-screen interface to gradually encourage students to increase their range of movement by allowing them to reach for more distant buttons and input controls.

\section{Future Work}

The SHIVA software prototypes provided a relatively small subset of modelling features which limits the range of objects which can be created with the system. A more flexible system would be desired for more advanced users (children and adults), though it is unclear which modelling paradigms would be the most suitable for disabled access.

The SHIVA GUI represented a state-of-the-art prototype system, but there were a number of issues and further needs resulting from the project. These include: the system had a heavy requirement on technical support staff for profile creation, which was stored in the XML format; automatic user adaptation was identified as important because it would help adjust to the user's needs; support for further input modalities: brain computer interface, gesture, multi-touch (including gesture interpretation and for collaborative input), tablet, and more general and complete eye-gaze support. 


\section{Acknowledgements}

We would like to thank the staff and students of Victoria Education Centre for their support of this project, as well as our colleagues for helping with the writing of this paper. The SHIVA project was funded by the EU INTERREG IV Two-Seas Programme.

\section{References}

Colven D. \& Judge S., (2006) Switch Access to Technology, ACE Centre Advisory Trust, Oxford, UK.

Fryazinov O. \& Pasko A. (2008) Interactive ray shading of FRep objects. In: WSCG' 2008, Communications Papers proceeding, Plzen - Bory, Czech Republic, pp. 145-152

Pasko A., Adzhiev V., Sourin A., \& Savchenko V. (1995) Function representation in geometric modeling: concepts, implementation and applications. The Visual Computer 11(8), 429-446

A. Pasko, V. Adzhiev. (2004) Function-based shape modeling: mathematical framework and specialized language, Automated Deduction in Geometry, Lecture Notes in Artificial Intelligence 2930, SpringerVerlag, pp. 132-160.

Sanchez M., Fryazinov O., \& Pasko A. (2012) Efficient evaluation of continuous signed distance to a polygonal mesh. In: Proceedings of SCCG 2012 (pp. 101-108)

Sanchez M., Fryazinov O., Vilbrandt T., \& Pasko A. (2013) Morphological shape generation through usercontrolled group metamorphosis. Computers \& Graphics 37(6): 620-627 